\title{
Exercise composition: from environment properties to composed problems
}

\author{
Isabel Araújo ${ }^{1}$, José João Almeida ${ }^{2}$, and Georgi Smirnov ${ }^{1}$ \\ ${ }^{1}$ Departamento de Matemática e Aplicações, Universidade do Minho \\ 2 Departamento de Informática, Universidade do Minho \\ isaraujo@iol.pt, jj@di.uminho.pt, smirnov@math.uminho.pt
}

\begin{abstract}
It is well known that (1) problems have a structure, (2) often this structure comes from its input, output or internal objects, (3) this structure organizes the problem resolution. Decomposition of a problem by its structure is a typical path in problem resolution.

In this article we will do the inverse: composition of simple problems using structure-builder operators.

We will start by (1) discuss problem structures, (2) select a set of important structure-builder operators, (3) study their properties, (4) apply them to a simple math case-study.
\end{abstract}

\section{Introduction}

Problems have a structure, usually connected with its input, output or internal objects.

With different names and flavors, problem decomposition was recognized as a crucial step in mathematics, physics and philosophy since the ancient times.

One incredible 4000 years old Egyptian papyrus ${ }^{1}$ claimed that (many) problems can be seen as composition of "chip's part problems", "aha problems" 2 or

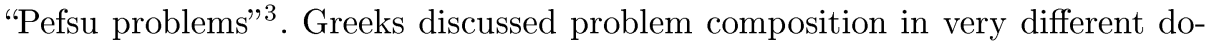
mains. Descartes included this subject in the second principle of Discourse on the Method.

Today it continues to be a rather challenging task in a wide number of areas.

The study of the problem structures plays a central role in mathematics and computer science areas $[3,7]$.

Some problems can be described as (1) pairs, triples, tuples of problems (p1,p2,p3), (2) functional composition - p3(p2(p1())), (3) systems of equation problems - (union of constraints), (4) inductive problems - (sequences, trees), and that internal structure can be used to decompose them in smaller problems. We will discuss some common problem structures in next section.

\footnotetext{
${ }^{1}$ See Wikipedia, Moscow Mathematical Papyrus

${ }^{2}$ Aha stands for a variable $-x$ in modern math notation.

${ }^{3}$ A pefsu measures the strength of the beer made from a heqat of grain.
} 


\section{Problem structures and exercise generation -}

Studying the problem structure is a very important subject because: (1) it helps in problem resolution, $(a)$ we can decompose the problem - divide and conquer approaches, $(b)$ we are able to transform the problem into a description where reusable patterns are easier to see; (2) it helps in problem understanding.

In a complementary way, problem structure can be used to assist in problem composition and exercise generation[1,9]. Beside the main problem text, in the area of exercise generation it is necessary to produce the exercise resolution, the suggestions, the results, the verification functions [5], metadata, etc. Problem decomposition and problem structure may guide in the construction of the exercise components. For example, sub-problems: (1) can be used as sub-questions; (2) may organize the generation of resolutions; (3) are crucial in the generation of suggestions; (4) can be used to organize problem recommendation.

\section{Problem structure and didactic strategy -}

Problem structure may also be used to systematically present different didactic choices to apply to different kinds of students or different situations. Example:

- ask for sub-problems before asking for the full problem,

- ask for the full problem and suggest sub-problems if necessary,

- incremental strategy.

\section{A simple example -}

The following example is presented to briefly illustrates some of the notions previously presented.

Problem 1: Consider figure bellow. Calculate the area of a rectangle measuring $5 \mathrm{~m}$ by $10 \mathrm{~m}$ with a circular hole with diameter $3 \mathrm{~m}$.
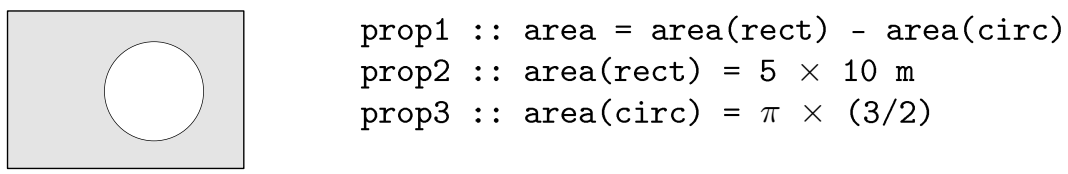

$$
\begin{aligned}
& p 1=\text { prop } 2:: \text { a,b } \rightarrow \text { area }(\text { rect }) \\
& p 2=\text { prop } 3:: \text { diameter } \rightarrow \text { area }(\text { circ }) \\
& p 3=p r o p 1:: \text { area }(\text { rect }), \text { area }(\text { circ }) \rightarrow \text { area }(\text { gray }) \\
& p 4=p 3 \circ(p 1, p 2):: a, b, \text { diameter } \rightarrow \text { area }(\text { gray })
\end{aligned}
$$

Problem structure: $\mathrm{p} 1(\mathrm{p} 2, \mathrm{p} 3)$.

Sub-problem order: (p2 before $\mathrm{p} 1)$; (p3 before $\mathrm{p} 1$ ).

Suggestions that can be provided to students:

1. tips to help in the problem decomposition: "start by calculate area(rect)" or "start by calculate area(circ)"; 